\title{
Insetos como Bioindicadores de Áreas Degradadas ou em Processo de Restauração no Bioma Caatinga
}

\section{Insect as Degraded Areas Bioindicators or in Restoration Process in the Caatinga Biome}

\author{
Ariane Costa Agra*a; Welber da Costa Pina ${ }^{\mathrm{b}}$ \\ ${ }^{a}$ Universidade Estadual de Santa Cruz, Programa de Pós-Graduação Stricto Sensu em Biologia e Biotecnologia de Microrganismos. BA, Brasil. \\ ${ }^{b}$ Universidade do Estado da Bahia. BA. Brasil. \\ *E-mail: arianeagra@gmail.com
}

\begin{abstract}
Resumo
O bioma Caatinga tem sofrido com grandes processos de degradação ambiental, que leva a fragmentação, perda do habitat e a uma crescente preocupação por uma restauração deste ambiente. Os insetos respondem de diferentes maneiras ao habitar gradientes sucessionais, sendo importantes na detecção de ambientes alterados. Por isso, o objetivo foi construir uma pesquisa bibliográfica acerca dos estudos desenvolvidos na Caatinga com foco na utilização dos insetos em avaliação de processos de restauração, regeneração ou sucessão de áreas degradadas neste bioma. O acesso à bibliografia foi feito eletronicamente, buscou-se por artigos científicos, no portal Google Acadêmico e de periódicos da Coordenação de Aperfeiçoamento de Pessoal de Nível Superior - CAPES, as buscas foram feitas entre 12/06/17 a 10/09/17 e em abril de 2020. As palavras chaves utilizadas foram em inglês e português e com relação ao tema proposto. Dos 112 artigos selecionados, apenas 18 estudos foram foco da revisão. A ordem de inseto mais estudada em áreas degradadas e/ou processo de regeneração é Isoptera, seguidos pela ordem Coleoptera e Hymenoptera. Todos os estudos apontaram grupos de insetos como bioindicadores, mas os resultados sobre assembleias, composição e riqueza de espécies foram divergentes para determinados grupos. Os trabalhos são pontuais em algumas ordens de insetos, mas aparentemente estão em legítima expansão e, assim, o bioma carece urgentemente de um maior aporte de pesquisas científicas, que permitirá um maior entendimento dos processos que catalisam a regeneração da vegetação nativa e a melhoria na capacidade de manejo de áreas degradadas.
\end{abstract}

Palavras-chave: Degradação Ambiental. Insetos da Caatinga. Restauração Ambiental.

\begin{abstract}
The Caatinga biome has undergone major processes of environmental degradation, which leads to fragmentation, loss of habitat and a growing concern for the restoration of this environment. Insects respond in different ways to inhabit successional gradients, being important in detecting altered environments. Therefore, the objective was to build a bibliographic research about the studies developed in the Caatinga with a focus on the use of insects in the evaluation of restoration, regeneration or succession of degraded areas in this biome. Access to the bibliography was carried out electronically, scientific articles were searched on the google academic and of periodics of the Coordination for the Improvement of Higher Education Personnel - CAPES, the searches were made between 06/12/17 to 09/10/17 and in April 2020. The keywords used were in English and Portuguese and in relation to the proposed theme. Of the 112 selected articles, only 18 studies were the focus of the review. The most studied insect order in degraded areas and / or regeneration processes is Isoptera, followed by Coleoptera and Hymenoptera. All studies pointed out groups of insects as bioindicators, but the results on assemblages, composition and species richness were divergent for certain groups. The works are punctual in some orders of insects, but it seems that they are in legitimate expansion and, thus, the biome urgently needs a greater contribution of scientific research, which will allow a greater understanding of the processes that catalyze the regeneration of native vegetation and improve the management capacity of degraded areas.
\end{abstract}

Keywords: Environmental Degradation. Caatinga Insects. Environmental Restoration.

\section{Introdução}

O bioma Caatinga ocupa a maior parte da região semiárida do brasil e representa aproximadamente $10 \%$ do território nacional (cerca de $800.000 \mathrm{~km}^{2}$ ) (MMA, 2009). O domínio das Caatingas é composto por uma grande diversidade de fitofisionomias, variando de florestas arbóreas ou arbustivas até áreas com vegetação esparsa (PRADO et al., 2005). Quando comparada com outras regiões semiáridas do mundo, a Caatinga se destaca por ter alta riqueza de espécies e elevado grau de endemismo (LEAL et al., 2005a, 2005b; RIBEIRO et al., 2015).

As principais fontes de degradação da Caatinga são associadas a agricultura, produção de carvão natural (lenha), a mineração e a pecuária (LEAL et al., 2005b). Esses impactos associados com o aumento das secas e a diminuição da precipitação média anual, decorrentes das mudanças climáticas, são proponentes do processo de desertificação que afeta grandes áreas do bioma (BRASIL, 2007; INSA, 2011). Além disso, áreas de Caatinga que foram abandonadas após atividades de agricultura intensiva apresentam baixa taxa de regeneração, podendo levar décadas para retornar a sua vegetação (PEREIRA et al., 2003). Consequência disso é a fragmentação de habitat, a invasão de espécies exóticas, a perda de espécies e de processos ecológicos importantes para manutenção dos serviços ambientais neste bioma (LEAL et al., 2005b; IBGE, 2018).

Sabe-se que os insetos compõem o grupo de animais 
mais numerosos do globo terrestre, com grande densidade populacional e representação em ampla distribuição geográfica. Os insetos são considerados fundamentais para a manutenção do funcionamento do ecossistema (LEWINSOHN et al., 2005). As mudanças na abundância de insetos indicam disponibilidade de recursos, alteração no habitat ou em seus inimigos naturais (BROWN, 1997). Estudos de diversidade faunística nos trópicos e, particularmente, para insetos na Caatinga indicaram que as taxas atingem indicadores mais elevados de diversidade em ambientes com estrutura de vegetação mais complexa (LEAL, 2003). Assim, as comunidades de insetos respondem de diferentes maneiras ao habitar gradientes sucessionais e/ou diferentes limites especiais de verticalidade (HUMPHREY et al., 1999; LEAL, 2003). Contudo, ainda não está claro, como as comunidades de insetos mudam nestes gradientes em sistemas secos tropicais, incluído o bioma Caatinga (NEVES et al., 2010b). Portanto, é necessário um conhecimento detalhado do ecossistema, caracterizando o nível de uso e manipulação do meio ambiente, para que não perca sua capacidade de recuperação após a realização das intervenções (AVILA et al., 2015)

A Caatinga vem sofrendo, ao longo dos anos, uma crescente deterioração dos diversos habitats naturais decorrentes, principalmente, de ações antrópicas. A escassez de informações científicas circundantes sobre o bioma, contribui para estágios mais acelerados de degradação e permanência da percepção de menosprezo da região em comparação com outras do país. Assim, é de suma importância oferecer elementos capazes de fundamentar argumentos, influenciar atitudes e contribuir para a escolha de encaminhamentos adequados, levando em consideração as necessidades das espécies locais e dos outros componentes ambientais elementares para a vida. Além disso, essa revisão sobre o Bioma Caatinga, representa uma grande contribuição na construção de conhecimento para as ciências, do ainda pouco explorado e pesquisado ambiente, carente de referencial teórico que lhe dê a visibilidade necessária e condizente com sua relevância social, econômica e ambiental.

Em virtude disso, o objetivo deste trabalho foi construir uma pesquisa bibliográfica acerca de estudos desenvolvidos na Caatinga com foco na utilização dos insetos como bioindicadores em avaliação de áreas degradadas e/ou em processos de restauração, regeneração ou sucessão. Ademais, verificar o estado da arte acerca do tema proposto.

\section{Desenvolvimento}

\subsection{Metodologia}

Foi realizado um estudo descritivo através de uma pesquisa bibliográfica. A preocupação com a pesquisa foi para avaliar as publicações pertinentes ao tema, de forma a categorizar o que é de fato relevante. Nesse sentido, a problemática em questão está relacionada à garantia de que a escolha da bibliografia tenha sido feita dentro de um universo de estudo que consiga de fato representar o estado da arte, além de primar pela qualidade, abrangência e significância das pesquisas.

$\mathrm{O}$ acesso à bibliografia foi feito eletronicamente, buscou-se somente por artigos científicos, no portal Google Acadêmico (http://www.scholar.google.com.br) e no portal de periódicos da Coordenação de Aperfeiçoamento de Pessoal de Nível Superior - CAPES. As buscas dos artigos foram realizadas em dois períodos: o primeiro entre 12/06/17 a 10/09/17 e o segundo durante abril de 2020. As seguintes palavras-chave foram utilizadas: Tropical Dry Forest Restoration; Caatinga Biome Restoration; Caatinga Biome Regeneration; Caatinga Biome Degradation; Insecta Biome Caatinga Restoration/ Regeneration. Optou-se por utilização de palavras em inglês em virtude desta língua ser universal e, ao menos, os resumos devem ser publicados neste idioma nas revistas, possibilitando assim uma busca mais precisa.

Para cada palavra-chave, foram abertas as dez primeiras páginas sugeridas na busca. Os artigos eram selecionados caso as palavras-chave aparecessem no título ou no objetivo do trabalho, obviamente, sempre relacionado ao estudo dos insetos. A partir desses critérios, foi conduzida uma leitura dos artigos selecionados, onde identificou-se as informações e os dados contidos no material, bem como verificou-se as relações existentes entre eles de modo a analisar a sua consistência com o tema proposto.

É importante ressaltar que o processo de recuperação é descrito como restituição de um ecossistema ou de uma população silvestre degradada a uma condição não degradada, que pode ser diferente de sua condição original, enquanto o processo de restauração consiste na restituição de um ecossistema ou de uma população silvestre degradada o mais próximo possível da sua condição original (REIS et al., 2003). Apesar dessa definição, na prática estes termos ainda se sobrepõem. Durante o desenvolvimento deste trabalho será sempre utilizado o termo recuperação, quando a intenção da recomposição do ecossistema original não estiver explicitada nas práticas citadas. Quando for feita referência aos artigos, o texto original será mantido.

\subsection{Resultados e Discussão}

Combinando-se todas variáveis de busca, foram filtrados 317 trabalhos e selecionados 112 artigos para análise. Dos artigos selecionados, $84 \%(n=94)$ eram referentes a riqueza e diversidade de invertebrados e/ou artrópodes na Caatinga e somente $16 \%(n=18)$ dos artigos focavam em avaliar os insetos como bioindicadores de áreas degradadas e/ ou em processos de restauração, regeneração ou sucessão no bioma.

Dos 18 artigos, foco principal desta revisão, exceto a pesquisa de Bandeira et al. (2003), todos as demais são publicações recentes, há menos de uma década. A ordem de inseto mais estudada em áreas degradas e/ou processo de regeneração é Isoptera com quatro estudos, seguido pela ordem Coleoptera com três trabalhos (Quadro1). 
Quadro 1 - Ordens de insetos, os autores e o ano de publicação de pesquisas realizadas em área degradas e/ou em processo de restauração no bioma Caatinga

\begin{tabular}{|c|c|}
\hline $\begin{array}{c}\text { Ordem do } \\
\text { Inseto }\end{array}$ & Autor e Ano \\
\hline Isoptera & $\begin{array}{l}\text { Bandeira et al., (2003); Vasconcellos et al., } \\
\text { (2010); Alves et al. (2011) e Vianna Junior et } \\
\text { al., (2014). }\end{array}$ \\
\hline Coleoptera & $\begin{array}{l}\text { Neves et al. (2010a); Liberal et al., (2011) e } \\
\text { Vieira, Silva e Louzada (2017) }\end{array}$ \\
\hline Hymenoptera & $\begin{array}{l}\text { Neves et al., (2010b) e Sousa- Souto et al., } \\
(2016) \text {. }\end{array}$ \\
\hline Hemiptera & Creão-Duarte et al., (2016). \\
\hline $\begin{array}{l}\text { Entomofauna } \\
\text { (insetos em } \\
\text { geral) }\end{array}$ & $\begin{array}{l}\text { Carvalho-Fernades et al., (2012); Neves et al., } \\
\text { (2014); Sousa-Souto et al., (2014); Alves et al., } \\
\text { (2017); Lima et al., (2017); Brito et al., (2018); } \\
\text { Maia et al., (2019) e Matos et al., (2019). }\end{array}$ \\
\hline
\end{tabular}

Este resultado, apontando Isoptera como a mais estudada, foi uma surpresa. Esperava-se mais pesquisas com ordens de insetos mais conhecidas, populares, abundantes e diversas, como Coleoptera, Lepidoptera e Hymenoptera. Uma possível explicação para isto é que a maioria dos estudos tem direcionado para qualidade do solo em áreas degradadas, e os cupins parecem ser o foco para responder como bioindicadores (BANDEIRA et al., 2003; VASCONCELLOS et al., 2010; ALVES et al., 2011; VIANNA JUNIOR et al., 2014).

Entretanto, apesar de todos os estudos apontarem alguns grupos de cupins como bioindicadores, os resultados sobre assembleias, composição e riqueza de espécies foram divergentes. Para Bandeira et al. (2003), Vasconcellos et al. (2010) e Vianna Junior et al. (2014) áreas com vegetação aberta ou que sofreram perturbações ecológicas (fracas ou intensas) apresentam menor riqueza de espécies, menor diversidade e composição estrutural diferentes das apresentadas nas áreas não perturbadas. Porém, Alves et al. (2011) não encontraram suporte estatístico para fazer tais generalizações nas áreas estudadas. Este último estudo, ressalta que a ausência de diferenças nos padrões analisados entre as áreas é reflexo da similaridade das variáveis físicas ambientais consideradas em cada local de estudo.

A ordem Coleoptera, mais especificamente a família Scarabaeidae, foi objeto de três estudos. Apesar dos trabalhos terem utilizado a mesma metodologia e possuírem objetivos similares: avaliar as mudanças na abundância, riqueza e composição de espécies em áreas com diferentes usos da terra, os encontros foram divergentes. Enquanto para Neves et al. (2010a) a comunidade de escarabeídeos mudou ao longo de um gradiente sucessional na Caatinga estudada, com abundância, riqueza e composição das espécies sempre aumentando de estágio precoce para o estágio mais avançado de recuperação, Liberal et al. (2011) e Vieira, Silva e Louzada (2017) não encontraram diferenças significativas entre área perturbada e não perturbada. Liberal et al. (2011) sugeriram que o baixo grau de riqueza de espécies pode ter dificultado a compreensão da influência das mudanças nessas comunidades, exceto de forma isolada para algumas espécies, enquanto Vieira, Silva e Louzada (2017) justificaram que as áreas analisadas estavam se regenerando há 14 anos, consequentemente, assumindo o status de uma sucessão secundária, fato que mantém os elementos adequados à assembleia de Scarabaeinae.

Contudo, todos os trabalhos com coleópteros apontaram esses insetos como bons indicadores ecológicos. Além disso, apontaram que a complexidade estrutural do habitat, a sazonalidade e a disponibilidade de recursos são fatores importantes na determinação da comunidade de besouro.

$\mathrm{Na}$ ordem Hymenoptera, só foram encontradas pesquisas com formigas. Tanto Neves et al. (2010b) quanto SousaSouto et al. (2016) testaram a influência de dois fatores: complexidade do habitat (referida como riqueza de espécies e densidade de árvores) e sazonalidade na mudança da diversidade de formigas em áreas de Caatinga, e descobriram que os fatores acima contribuem para o aumento da riqueza de espécies deste grupo, bem como para a modificação da sua composição ao longo do gradiente sucessional (áreas mais conservadas com mais diversidade e mais endemismo), com forte influência da estação chuvosa. Ademais, Sousa-Souto et al. (2016) amostraram dezessete espécies exclusivas de área de sucessão tardia, indicando que essas espécies poderiam ser bioindicadores de estágios avançados de regeneração florestal na Caatinga. Encontros similares e, também, indicando espécies bioindicadoras foram apontadas igualmente por Neves et al. (2010b).

Os insetos da família Membracidae, vulgarmente designados como soldadinhos ou viuvinhas foi objeto de apenas um estudo. De acordo com achados de Creão-Duarte et al. (2016), durante a estação chuvosa, os membracidas de diferentes estruturas de vegetação são relativamente semelhantes (mais de $60 \%$ de similaridade). Por outro lado, durante a estação seca são diferentes, onde as áreas degradadas e intermediárias têm uma similaridade muito baixa quando comparada com áreas mais preservadas. Ademais, a sazonalidade dos membracídeos na Caatinga parece estar intimamente relacionada ao ciclo de vida sazonal das plantas hospedeiras, indicando que as áreas preservadas mantiveram uma grande riqueza durante a estação seca, destacando a importância destas áreas para a manutenção da diversidade do táxon.

Oitos artigos estudaram a macrofauna e/ou artrópodes (incluindo insetos) em avaliação de áreas com alterações antrópicas. Assim, em geral, as assembleias de artrópodes são frequentemente estudadas para descrever a sucessão e restauração da comunidade (PIK et al., 2002; LONGCORE, 2003; SCHNELL et al., 2003), para avaliar o estado de conservação (RIBEIRO et al., 1998; BARROW et al., 2007), e abordar a distribuição de espécies ao longo dos gradientes ambientais (BACH, 1993; ANTVOGEL; BONN, 2001, DAVIS; SCHOLTZ, 2004) e distúrbios (LAWTON et al., 1998; EGGLETON et al., 2002).

Neves et al. (2014), entre outros objetivos, testaram se a 
comunidade dos insetos herbívoros variava em um gradiente sucessional e vertical em uma floresta tropical seca. Eles concluíram que a riqueza de insetos sugadores de seiva aumentou ao longo do gradiente sucessional em ambas as escalas espaciais estudadas e, ainda, que a composição da comunidade de insetos herbívoros de alimentação livre, associada ao dossel, mudou significativamente entre os estádios de sucessão inicial e avançado (intermediário-tardio).

Sousa-Souto et al. (2014) testaram a hipótese de que o aumento da riqueza e densidade das árvores que ocorrem ao longo da sucessão da Caatinga pode determinar um efeito em cascata sobre insetos e aranhas, aumentando sua riqueza e abundância e alterando a composição da sua espécie. E resultados indicaram que as mudanças na estrutura do habitat conduzem parcialmente uma cascata trófica envolvendo conjuntos de insetos arbóreos e aranhas, mas é evidente que as aranhas arbóreas têm baixa sensibilidade à disponibilidade de recursos ou qualidade do habitat.

Neves et al. (2014) e Sousa-Souto et al. (2014), concluíram afirmando que, como um todo, os achados indicavam que os estágios intermediários e tardios são semelhantes na composição, na riqueza e nas guildas de algumas espécies de insetos sugerindo que após aproximadamente 40 a 50 anos de regeneração natural, a comunidade de insetos destes grupo associados é aparentemente restaurado. Porém, para outros grupos (ex: herbívoros comedores de folhas) diferença entre os estágios intermediário e tardio, no entanto, indicam que as funções do ecossistema provavelmente levam mais tempo para se regenerar.

Lima et al. (2017) estudaram fauna edáfica (entre eles, os insetos) como bioindicadora da recuperação de áreas degradadas no bioma Caatinga. Este trabalho teve como objetivo avaliar o emprego da fauna edáfica como bioindicadora da qualidade do solo em áreas com e sem a adição de topsoil em plantios florestais para a recuperação de áreas de extração de recurso mineral, a pirraça. Os encontros apontam que a fauna do solo, incluindo os insetos, foi eficiente como bioindicador para monitorar a qualidade ecológica das áreas avaliadas no bioma da Caatinga. Além disso, a complexidade ecológica da fauna do solo nas áreas de floresta nativa foi maior que a encontrada nas plantações florestais, independentemente da adição ou não de topsoil. Entretanto, é recomendada a adição de topsoil em plantações florestais usadas para recuperação de áreas de Caatinga degradadas, uma vez que esta prática teve um impacto positivo na comunidade de fauna do solo, em comparação com áreas sem adição deste solo.

Alves et al. (2017) buscaram avaliar a meso e macrofauna edáficas, antes e depois da implantação das mudas de espécies nativas em áreas degradadas de Caatinga, utilizando a fauna do solo como bioindicadora de restauração. Esta pesquisa apontou que ocorreu um aumento na abundância e riqueza dos táxons da fauna do solo, principalmente no período chuvoso, demonstrando os benefícios da implantação de plantas nativas para a restauração da área de Caatinga antropizada. Além disso, reafirma a utilização dos insetos como bioindicadores de áreas degradadas.

Os insetos galhadores foram alvos de dois estudos no Bioma. Um realizado por Carvalho-Fernandes et al. (2012) e o outro por Brito et al. (2018) ambas pesquisas estudaram os efeitos das atividades antrópicas em comunidades de insetos galhadores em áreas de Caatinga, contudo os resultados foram divergentes. Carvalho-Fernandes et al. (2012) constataram uma riqueza maior de morfotipos de galhas nas áreas preservadas do que nas áreas intermediárias e antropizadas, enquanto Brito et al. (2018) encontraram uma riqueza de galhas maior nas áreas intermediárias quando comparadas às áreas preservadas e antropizadas. Porém, ambos estudos, ressaltaram que os insetos galhadores podem ser úteis na avaliação do impacto humano sobre o ambiente.

Por fim, os estudos mais recentes de Matos et al. (2019) e Maia et al. (2019), ambos objetivaram realizar um levantamento da diversidade de artrópodes ou macrofauna (incluindo insetos) do solo em área de Caatinga, onde foi adotado diferentes tipos de manejo florestal. Os dois trabalhos apresentaram resultados similares, onde a comunidade edáfica é influenciada pelas diferentes etapas de exploração da madeira na área, sendo que o corte seletivo, que é um manejo mais conservador da Caatinga, favorece a manutenção do número de indivíduos e grupos de invertebrados.

Além disso, Maia et al. (2019) acrescentou que a sucessão de períodos de pousio contribuiu para o estabelecimento e reestruturação dos grupos de artrópodes, demonstrando a viabilidade de um ciclo de 10 anos de exploração de madeira na Caatinga analisada. Já Matos et al. (2019) incrementaram afirmando que o corte seletivo por espécies se mostrou menos impactante para a macrofauna do solo, devido aos valores mais expressivos de diversidade e índice de abundância e à ocorrência de organismos mais exigentes em condições de microclima, que caracterizam áreas preservadas. Ambos autores, destacaram em suas pesquisas a importância dos insetos na detecção de mudanças ambientais na Caatinga.

De fato, a fauna de artrópodes é normalmente muito diversa e abundante nos ambientes, por isso desperta a atenção dos pesquisadores. As abordagens dos artigos, em geral, são de avaliações de assembleias de grupo supraespecíficos. Essa abordagem pode economizar tempo e recursos (WARD; LARIVIÈRE, 2004), entretanto as assertivas são verdadeiras apenas quando espécies individuais dentro de cada táxon apresentarem respostas semelhantes aos gradientes ambientais. Além disso, no caso da Caatinga, um bioma altamente diverso e pouco explorado, o ideal é trabalhar com resoluções taxonômicas menores, menos abrangentes, como sugerido por Olsgard e Sommerfeld (2000) e Báldi (2003) para ambientes inexplorados.

Apesar da longa história de degradação ambiental no Brasil e, também, em outras partes do mundo, apenas recentemente tem sido desenvolvida linhas de pesquisa para entender a dinâmica desses processos e tentar revertê-las. Com isso, de 
acordo com Hobbs et al. (2011) a Ecologia da Restauração, juntamente com atividades relacionadas, é considerada um elemento chave para a conservação dos recursos naturais por meio da intervenção em ecossistema degradado e que tem ganhado atenção no gerenciamento de políticas públicas nas últimas décadas.

Assim, diante do imenso desafio da restauração em larga escala no Brasil, há uma demanda urgente por informações de ambientes menosprezados e pouco estudados, como a Caatinga, uma vez que ajudarão os tomadores de decisão a empreenderem projetos de restauração nos anos seguintes. Para uma maior compreensão e de uma visão geral das tendências de restauração ecológica nos biomas brasileiros, aconselho a leitura de Guerra et al. 2020 que trata de uma revisão sistemática da pesquisa publicada, a fim de apoiar políticas públicas, legislação, práticas de implementação aprimoradas e programas de ampliação.

\section{Conclusão}

Portanto, a presente pesquisa permitiu ampliar o conhecimento sobre o Bioma Caatinga e demonstrou que os insetos são bons bioindicadores ambientais de áreas com perturbações antrópicas, porém não é possível afirmar um padrão para a influência da alteração do habitat para a comunidade de insetos da Caatinga. Esta revisão mostrou ainda que, os artigos são pontuais e em poucas ordens de insetos, porém os estudos parecem em legítima expansão. Além disso, é importante destacar que o bioma carece urgentemente de um maior aporte de pesquisas científicas, que permitirá um maior entendimento dos processos que catalisam a regeneração da vegetação nativa e a melhoria na capacidade de manejo de áreas degradadas. Por fim, as descobertas fortalecem a ideia da importância dos habitats secundários para a manutenção da biodiversidade dos insetos.

\section{Referências}

ALVES, W.F. et al. Termites as bioindicators of habitat quality in the Caatinga, Brazil: is there agreement between structural habitat variables and the sampled assemblages? Neotrop. Entomol., v.40, n.1, p.39-46, 2011. doi: 10.1590/S1519-566X2011000100006

ANTVOGEL, H.; BONN, A. Environmental parameters and microspatial distribution of insects: a case study of carabids in an alluvial forest. Ecography, v.24, n.4, p.470-482, 2001. doi: 10.1111/j.1600-0587.2001.tb00482.x

AVILA, A.L.D. et al. Medium-term dynamics of tree species composition in response to silvicultural intervention intensities in a tropical rain Forest. Biol. Conserv., v.191, p.577-586, 2015. doi: 10.1016/j.biocon.2015.08.004

BACH, C.E. Effects of microclimate and plant characteristics on the distribution of a willow flea beetle, Altica Subplicata. Am. Midland Naturalist, v.130, n.1, p.193-208, 1993. doi: $10.2307 / 2426287$

BÁLDI, A. Using higher taxa as surrogates of species richness: a study based on 3700 Coleoptera, Diptera, and Acari species in Central-Hungarian reserves. Basic Appl. Ecol., v.4, n.6, p.589593, 2003. doi: 10.1078/1439-1791-00193
BANDEIRA, A.G. et al. Effects of habitat disturbance on the Termite fauna in a highland humid forest in the Caatinga domain, Brazil. Sociobiology, v.42, n.1, p.1-11, 2003.

BARROW, L.; PARR, C.L.; KOHENA, J.L. Habitat type influences fire resilience of ant assemblages in the semi-arid tropics of Northern Australia. J.Arid Environ., v.69, n.1, p.80-95, 2007. doi: 10.1016/j.jaridenv.2006.08.005

BRASIL. Ministério do Meio Ambiente. Braziliam forests at a glance-Reference Period: 2005-2009. Brasília: MMA, 2009.

BRASIL. Ministério do Meio Ambiente. Atlas das áreas susceptíveis á desertificação do Brasil. Paraíba: Secretaria de Recursos Hídricos e Universidade Federal da Paraíba, 2007.

BRITO, G.P. et al. Riqueza de galhas de insetos em áreas de Caatinga com diferentes graus de antropização do estado da Bahia, Brasil. Iheringia, Sér. Zool. v.108, p.1-9, 2018. doi: 10.1590/1678-4766e2018003

BROWN, K.S. Diversity, disturbance, and sustainable use of neotropical forests: insects as indicators for conservation monitoring. J. Insect Conserv. v.1, p.25-42, 1997.

CARVALHO-FERNANDES, S.P.; ALMEIDA-CORTEZ, J.S.; FERREIRA, A.L.N. Riqueza de galhas entomógenas em áreas antropizadas e preservadas de caatinga. Rev. Árvore, v.36, n.2, p.269-277, 2012. doi: 10.1590/S0100-67622012000200008

CREÃO-DUARTE,A.J. et al. Temporal variation of Membracidae (Hemiptera: Auchenorrhyncha) composition in areas of Caatinga with different vegetation structures. Sociobiology, v.63, n.2, p.826-830, 2016. doi: 10.13102/sociobiology.v63i2.1071

DAVIS, A.L.; SCHOLTZ, C.H. Local and regional species ranges of a dung beetle assemblage from the semi-arid Karoo/Kalahari margins, South Africa. J. Arid Environ., v.57, n.1, p.61-85, 2004. doi: 10.1016/S0140-1963(03)00094-6

EGGLETON, P. et al. Termite diversity across an anthropogenic disturbance gradient in the humid forest zone of West Africa. Agric., Ecosystems Environ., v.90, n.2, p.189-202, 2002. doi: 10.1016/S0167-8809(01)00206-7

GUERRA, A. et al. Drivers and projections of vegetation loss in the Pantanal and surrounding ecosystems. Land Use Policy, v.91, 2020. doi: 10.1016/j.landusepol. 2019.104388.

HOBBS, R.J. et al. Intervention ecology: applying ecological science in the twenty-first century. Bioscience, v.61, p.442-450, 2011. doi: 10.1525/bio.2011.61.6.6

HUMPHREY, J.W. et al. Relationships between insect diversity and habitat characteristics in plantation forests. Forest Ecol. Manag., v.113, p.11-21, 1999. doi: 10.1016/S03781127(98)00413-7

IBGE - Instituto Brasileiro de Geografia e Estatística Biomas continentais do Brasil. Disponível em: https://concla.ibge.gov.br/ images/pdf/vamoscontar/texto_biomas.pdf. Acesso em: 2 maio 2020.

INSA - Instituto Nacional do Semiárido. Desertificação e mudanças climáticas no Semiárido Brasileiro. Campina Grande: INSA, 2011.

LAWTON, J.H. et al. Biodiversity inventories, indicator taxa and effects of habitat modification in tropical forest. Letters Nat., v.391, n.1, p.72-76, 1998. doi: 10.1038/34166

LEAL, I.R.; TABARELLI, M.; SILVA, J.M.C. Ecologia e Conservação da Caatinga. Recife: Universitária da Universidade Federal de Pernambuco, 2005a.

LEAL, I.R. et al. Changing the course of biodiversity conservation in the Caatinga of Northeastern Brazil. Conserv. Biol., v.19, 
p.701-706, 2005b. doi: 10.1111/j.1523-1739.2005.00703.x

LEAL, I.R. Diversidade de formigas em diferentes unidades de paisagem da caatinga. In: LEAL, I.R.; TABARELLI, M.; SILVA, J.M.C. (Ed.). Ecologia e conservação da caatinga. Recife: Universitária da Universidade Federal de Pernambuco, 2003. p.435-461

LEWINSOHN, T.M.; NOVOTNY, V.; BASSET, Y. Insects on plants: diversity of herbivore assemblages revisited. Ecol. Evolution Syst., v.36, p.597-620, 2005. doi: 10.1146/annurev. ecolsys.36.091704.175520.

LIBERAL, C.N. et al. How habitat change and rainfall affect dung beetle diversity in Caatinga, a Brazilian semi-arid ecosystem. $J$. Insect Scie., v.11, n.114, p.1-11, 2011. doi: 10.1673/031.011.11401

LIMA, K.D.R. et al. Soil fauna as bioindicator of recovery of degraded areas in the Caatinga biome. Rev. Caatinga, v.30, n.2, p.401-411, 2017. doi: 10.1590/1983-21252017v30n215rc

LONGCORE, T. Terrestrial Arthropods as indicators of ecological restoration success in coastal sage scrub (California, U.S.A.). Restoration Ecol., v.11, n.4, p.397-409, 2003. doi: 10.1046/j.1526-100X.2003.rec0221.x

MAIA, L.S. et al. Entomofauna diversity in areas of Caatinga under forest management in the semi-arid region of Ceará. Comunicata Scie., v.10, n.1, p.10-20, 2019. doi: 10.14295/ cs.v10i1.2422.

MATOS, P.S.; BARRETO-GARCIA, P.A.B.; SCORIZA, R.N. Effect of different forest management practices on the soil macrofauna in the arboreal caatinga. Rev. Caatinga, v.32, n.3, p.741-750, 2019. doi: 10.1590/1983-21252019v32n318rc

NEVES, F.S. et al. Successional and seasonal changes in a community of dung beetles (Coleoptera: Scarabaeinae) in a Brazilian tropical dry forest. Nat. Conserv., v.8, n.2, p.160-164, 2010a. doi: 10.4322/natcon.00802009

NEVES, F.S. et al. Diversity of arboreal ants in a brazilian tropical dry forest: effects of seasonality and successional stage. Sociobiology, v.56, p.1-18, 2010 b.

NEVES, F.S. et al. Insect herbivores and leaf damage along successional and vertical gradients in a tropical dry forest. Biotropica, v.46, n.1, p.14-24, 2014. doi: 10.1111/btp.12068

OLSGARD, F.; SOMERFIELD, P.J. Surrogates in marine benthic investigations: which taxonomic unit to target? $J$. Aquatic Ecosystem Stress Recovery, v.7, n.1, p.25-42, 2000. doi: 10.1023/A:1009967313147
PEREIRA, I.M. et al. Use-history effects on structure and flora of Caatinga. Biotropica, v.35, p.154-165, 2003. doi: 10.1111/j.17447429.2003.tb00275.x

PIK, A.J. et al. The use of invertebrates to detect small scale habitat heterogeinity and its application to restoration practices. Environ. Monitoring Assessment, v.75, n.2, p.179-199, 2002.

PRADO, D.E. et al. As Caatingas da América do Sul: ecologia e conservação da Caatinga. Recife: Universitária da Universidade Federal de Pernambuco, 2005.

REIS, A. et al. Restauração de áreas degradadas: a nucleação como base para os processos sucessionais. Nat. Conserv., v.1, p.28-36, 2003.

RIBEIRO, S.P.; CARNEIRO, M.A.A.; FERNANDES, W. Freefeeding insect herbivores along environmental gradients in Serra do Cipó: basis for a management plan. J. Insect Conservation, v.2, n.2, p.107-118, 1998. doi: 10.1023/A:1009669405776

SCHNELL, M.R.; PIK, A.J.; DANGERFIELD, J.M. Ant community succession within eucalypt plantations on used pasture and implications for taxonomic sufficiency in biomonitoring. Austral Ecology, v.28, n.5, p.553-565, 2003.

SOUSA-SOUTO, L. et al. Is there a bottom-up cascade on the assemblages of trees, arboreal insects and spiders in a semiarid Caatinga? Arthropod-Plant Interactions, v.8, p.581-591, 2014. doi: 10.1007/s11829-014-9341-0

SOUSA-SOUTO, L. et al. Composition and richness of arboreal ants in fragments of brazilian Caatinga: effects of secondary succession. Sociobiology, v.63, n.2, p.762-769, 2016. doi: 10.13102/sociobiology.v63i2.909

VASCONCELLOS, A. et al. Termite assemblages in three habitats under different disturbance regimes in the semi-arid Caatinga of NE Brazil. J. Arid Environment, v.74, p.298-302, 2010. doi: 10.1016/j.jaridenv.2009.07.007

VIANNA JUNIOR, A.B. et al. Termite assemblages in dry tropical forests of Northeastern Brazil: are termites bioindicators of environmental disturbances? Sociobiology, v.61, n.3, p.324331, 2014. doi: 10.13102/sociobiology.v61i3.324-331

VIEIRA, L.; SILVA, F.A.B.; LOUZADA, J. Dung beetles in a Caatinga Natural Reserve: a threatened Brazilian dry-forest with high biological value. Iheringia, Sér. Zool. v.107, p.1-6, 2017. doi: 10.1590/1678-4766e2017045

WARD, D.F.; LARIVIÈRE, M.C. Terrestrial invertebrate surveys and rapid biodiversity assessment in New Zeland: lessons from Australia. New Zealand J. Ecol., v.28, n.1, p.151-159, 2004. 